\title{
Regulating Positive and Negative Emotions in Daily Life
}

\author{
John B. Nezlek ${ }^{1}$ and Peter Kuppens ${ }^{2}$ \\ ${ }^{1}$ College of William and Mary \\ ${ }^{2}$ Katholieke Universiteit Leuven
}

\begin{abstract}
The present study examined how people regulate their emotions in daily life and how such regulation is related to their daily affective experience and psychological adjustment. Each day for an average of 3 weeks, participants described how they had regulated their emotions in terms of the reappraisal and suppression (inhibiting the expression) of positive and negative emotions, and they described their emotional experience, self-esteem, and psychological adjustment in terms of Beck's triadic model of depression. Reappraisal was used more often than suppression, and suppressing positive emotions was used less than the other three strategies. In general, regulation through reappraisal was found to be beneficial, whereas regulation by suppression was not. Reappraisal of positive emotions was associated with increases in positive affect, self-esteem, and psychological adjustment, whereas suppressing positive emotions was associated with decreased positive emotion, selfesteem, and psychological adjustment, and increased negative emotions. Moreover, relationships between reappraisal and psychological adjustment and self-esteem were mediated by experienced positive affect, whereas relationships between suppression of positive emotions and self-esteem adjustment were mediated by negative affect.
\end{abstract}

Emotions are central components of people's lives, both interpersonally and intrapersonally, and emotional experiences can have powerful impacts on people's functioning, both positive and negative.

Preparation of this paper was supported by KULeuven Research Council Grants GOA/05/04 and F/05/047 and by a Faculty Research Grant from the College of William and Mary. The second author is a postdoctoral fellow of the Fund for Scientific Research-Flanders.

Correspondence concerning this article should be addressed to John B. Nezlek, College of William and Mary, Department of Psychology, PO Box 8795, Williamsburg, VA, 23187-8795. E-mail: jbnezl@wm.edu.

Journal of Personality 76:3, June 2008

(C) 2008, Copyright the Authors

Journal compilation (C) 2008, Blackwell Publishing, Inc.

DOI: $10.1111 / \mathrm{j} .1467-6494.2008 .00496 . x$ 
Perhaps due to this strong impact, emotional experience is not free floating but is regulated to keep one's emotional experiences and expressions in check and to meet one's own and societies' demands. Considerable research indicates that the regulation of one's emotions contributes to various important outcomes. Such outcomes include decreased risk for depression (e.g., Davidson, Pizzagalli, Nitschke, \& Putnam, 2002), increased control over the expression of violence (e.g., Davidson, Putnam, \& Larson, 2000), a more developed sense of morality (e.g., Eisenberg, 2000), and improved psychological development (e.g., Dodge, 1989), among others.

Despite these broad and important consequences, little is known about how people regulate their emotions in their daily lives. Although valuable, much of the existing research has either conceptualized emotional regulation as a trait (an individual difference that is presumed to be relatively stable across time and situations, e.g., Gross \& John, 2003) or has examined regulation in controlled laboratory settings (e.g., in reaction to emotion-inducing stimuli such as film clips, Gross \& Levenson, 1997). Nevertheless, a full understanding of emotional regulation requires understanding the roles it plays in the ebb and flow of daily life. Moreover, much of the existing research on emotional regulation has not distinguished the regulation of positive and negative emotion whereas we believe there are good reasons to do so.

The present study was intended to complement and extend work on emotional regulation in two ways - by studying emotional regulation as a state that occurs in daily life and by distinguishing the regulation of positive and negative emotions. In particular, we examined how (in terms of frequency of use) individuals use different emotional regulation strategies in their daily life and how these strategies are associated with other aspects of psychological functioning such as changes in emotional experience and changes in psychological adjustment.

\section{Types of Emotion Regulation}

Our conceptualization of emotional regulation was based on the model proposed by James Gross (e.g., Gross, 1998). Gross distinguishes two strategies of emotional regulation: reappraisal and suppression. Reappraisal consists of changing the way a situation is construed so as to decrease its emotional impact. Suppression 
consists of inhibiting the outward signs of inner feelings. Existing research suggests that these two strategies have different outcomes and that only reappraisal decreases emotional experience (e.g., Gross, 2002). Furthermore, greater use of reappraisal has been found to be associated with beneficial outcomes such as greater psychological adjustment and improved interpersonal functioning, whereas greater use of suppression has been found to be negatively related to such outcomes (Gross \& John, 2003).

Gross's model, at least in terms of measuring regulation strategies at the trait level, does not distinguish the regulation of positive and negative emotions. Nevertheless, a growing body of research suggests that positive and negative emotions reflect the operation of systems that can be distinguished (e.g., Cacioppo \& Gardner, 1999) even to the point that the neural circuits comprising these two systems do not overlap completely (e.g., Panksepp, 1998). By implication, the regulation of positive and negative emotions may reflect distinct, albeit related, processes. For example, some experimental research suggests that suppressing the expression of positive emotions has an impact on people's emotional experiences, whereas suppressing the expression of negative emotions does not (e.g., Gross $\&$ Levenson, 1997). Such findings suggest that the effects of emotional regulation are not consistent across positive and negative emotions.

Therefore, we examined the use of four different emotional regulation strategies: reappraisal of positive and negative emotions and suppression of positive and negative emotions. First, we examined how often people use these strategies to regulate their emotions in daily life. Second, we examined how the use of such strategies was related to emotional experience. In this regard, we were concerned primarily with the strength and direction of the within-person covariation between emotional regulation and positive and negative emotional experience. Understanding such covariation addresses at least two important issues.

First, knowing the within-person relationships between regulation and emotional experience can provide some insight into how effective different regulatory strategies are in altering emotional experience. For example, it is not clear if people can regulate positive and negative emotions equally well. Negative emotions may be stronger than positive emotions and occur less frequently than positive emotions (e.g., Taylor, 1991), a combination that suggests they may be harder to regulate. Such a difference may be particularly 
pronounced for suppression-regulation that is not primarily aimed at changing experience but rather at the reduction of the expression of emotions.

Second, knowing the relationships between emotion-regulation strategies and emotional experience may indicate what types of emotion regulation become more likely when an individual is experiencing particular emotions. For example, certain forms of regulation might be easier or might be facilitated by specific emotions. There is evidence that emotional states in themselves affect appraisal tendencies (e.g., Lerner \& Keltner, 2001). Furthermore, the experience of particular emotions can affect the psychological resources needed to perform emotion regulation efforts. According to the broadenand-build model of positive emotions (Fredrickson, 2001), the experience of positive emotions broadens one's perspective and thought-action repertoire, which suggests that the experience of positive emotions may increase emotion-regulation capacities by facilitating reappraisal.

\section{Emotion Regulation, Psychological Adjustment, and Self-Esteem}

In addition to examining the occurrence of the regulation of positive and negative emotions and their relationships with emotional experience, we examined relationships between emotional regulation and psychological adjustment and between regulation and self-esteem. We defined psychological adjustment in terms of Beck's triadic model of depression (e.g., Beck, 1972). Previous research on such relationships has concerned only trait-level relationships. We sought to extend the focus of emotional regulation research by examining relationships between emotional regulation and psychological adjustment and self-esteem at the within-person level. Such a distinction is critical because relationships at the trait level may not tell us anything about relationships at the state level. Trait- and state-level relationships are mathematically independent (e.g., Nezlek, 2001), and they may represent meaningfully different processes (e.g., Tennen, Affleck, Armeli, \& Carney, 2000). On the basis of previous research (e.g., Gross \& John, 2003), our general expectation was that reappraisal would be related positively to psychological adjustment and self-esteem, whereas suppression would be negatively related.

Furthermore, considerable research at both the trait (e.g., Diener, Suh, Lucas, \& Smith, 1999) and state levels (e.g., Nezlek, 2005) 
indicates that psychological adjustment and self-esteem have strong affective components. Given this, it is reasonable to expect that regulating one's emotions is associated with adjustment and self-esteem because of its relationships with emotional experience. Therefore, we examined the extent to which relationships between daily regulation and daily adjustment and self-esteem were mediated by relationships between daily regulation and daily affect. Trait-level research (e.g., Gross \& John, 2003) suggests that emotional states do not mediate relationships between emotional regulation and psychological adjustment and self-esteem, i.e., relationships between trait emotional regulation and trait adjustment are direct. Nevertheless, as noted above, state (within-person) relationships may represent different processes than trait (between-person) relationships.

To address the issues raised above, the present study examined within-person relationships between emotional regulation and emotional experience and psychological adjustment and selfesteem in daily life. Each day for 3 weeks, participants described the emotional regulation strategies they used, described their emotional experiences, and provided measures of self-esteem and psychological adjustment. The analyses estimated within-person relationships between emotional regulation and the other daily constructs.

The analyses were guided by the following two broad hypotheses:

(1) Reappraisal will be associated with beneficial outcomes. Suppression will not.

(2) Positive emotions will be easier to regulate than negative emotions.

\section{METHOD}

Participants were 68 male and 85 female undergraduates who used the Internet to provide data at the end of each day for 3 weeks. Inspection of date and time stamps led to the deletion of some data, leaving 2,859 days of data $(M=18.7, S D=3.79)$. Data were deleted if they were provided before 8:00 p.m. of the day in question or past 7:00 a.m. of the next morning. The minimum number of days a participant contributed data was 6 , and the maximum was 28 . Only 11 participants provided fewer than 14 days of data, and only 18 provided more than 22 days of data. It should be noted that the multilevel modeling analyses we used 
take into account the numbers of observations a unit of analysis contributes as part of what is called "precision weighting" (Raudenbush \& Bryk, 2002).

The daily measures included a four-item measure of self-esteem (Items 3, 6, 7, and 10 of the Rosenberg Self-Esteem scale; Rosenberg, 1965) reworded for daily administration. Daily psychological adjustment was measured by three items representing the elements of Beck's cognitive triad (Beck, 1972): (1) negative view of self, "Overall, how positively did you feel about yourself today"; (2) negative view of life in general, "Thinking of your life in general, how well did things go today"; and 3) negative view of the future, "How optimistic are you about how your life (in general) will be tomorrow." A more detailed description of these measures can be found in Nezlek (2005).

Daily affect was measured following a circumplex model (e.g., Feldman, Barrett, \& Russell, 1998). Positive active affect (PA) was measured with the following items: enthusiastic, happy, active, energetic, alert, proud, joyful, and happy. Positive deactive affect (PD) was measured with the items calm, satisfied, and relaxed. Negative active affect (NA) was measured with guilty, nervous, afraid, angry, ashamed, embarrassed, upset, and disgusted. Negative deactive affect (ND) was measured with sluggish, sad, tired, bored, and sleepy. All responses were made on 7-point scales for which higher numbers meant more strongly or more so.

In addition, participants provided daily measures of emotional regulation based on the measure described by Gross and John (2003). We chose two items (one positive and one negative) from both the reappraisal and suppression scales based on factor loadings and appropriateness for daily administration. Items from this trait-level measure were reworded slightly to make them appropriate for daily administration. Reappraisal was measured with the following items: "When I wanted to feel a more positive emotion (such as happiness or amusement), I changed what I was thinking about," and "When I wanted to feel less negative emotion, I changed what I was thinking about." Suppression was measured with the following items: "When I was feeling positive emotions, I was careful not to express them," and "When I felt negative emotions (such as sadness, nervousness, or anger), I was careful not to express them." To ensure that participants responded to these questions in terms of how they felt that day, each of these items was preceded by the stem "Answer each question about what you did or how you felt today," and participants answered using a 1-7 scale with endpoints labeled "not at all characteristic of me" and "very characteristic of me." Moreover, because participants were answering these questions as part of a daily measure, we felt that the combination of the scale points and the context clearly requested a state rather than a trait rating. 
Table 1

Descriptive Statistics for Daily Measures

\begin{tabular}{|c|c|c|c|c|}
\hline & Mean & $\begin{array}{l}\text { Vithin-Person } \\
\text { Variance }\end{array}$ & $\begin{array}{c}\text { Between-Person } \\
\text { Variance }\end{array}$ & Reliability \\
\hline Positive reappraisal & 3.69 & 1.28 & 1.82 & \\
\hline Negative reappraisal & 3.62 & 1.30 & 1.69 & \\
\hline Positive suppression & 2.53 & 1.07 & 1.28 & \\
\hline Negative suppression & 3.37 & 1.74 & 1.78 & \\
\hline Positive active affect & 4.10 & .81 & .74 & .79 \\
\hline Positive deactive affect & 4.19 & .88 & .60 & .52 \\
\hline Negative active affect & 2.27 & .62 & .59 & .74 \\
\hline Negative deactive affect & 3.28 & .80 & .65 & .47 \\
\hline Cognitive triad & 5.34 & .69 & .64 & .75 \\
\hline Self-esteem & 5.51 & .68 & .78 & .62 \\
\hline
\end{tabular}

\section{Reliability of Daily Measures}

As discussed by Nezlek (2007), the day-level reliabilities for each of the daily measures that consisted of more than one item were estimated using a three-level model, items nested within days and days nested within persons. The estimates produced by these analyses are presented in Table 1 . Given these reliabilities, daily measures of these constructs were defined as the means for all the scale items.

\section{RESULTS}

The present data comprised a multilevel data structure because observations at one level of analysis (days) were nested within another level of analysis (people), and so the primary analyses were multilevel random coefficient models conducted using the program HLM (Raudenbush, Bryk, Cheong, \& Congdon, 2000). See Nezlek (2001) for a discussion of multilevel analyses of daily diary data. The analyses were two-level models, days nested within persons.

First, each of the daily measures was analyzed with a totally unconditional model, that is, no predictors at Level 1 or 2 . The analyses estimated how much of the total variance in each measure was within persons (Level 1, the state or day level) and how much was between persons (Level 2, the trait level). The results of these analyses are 
summarized in Table 1 . These analyses showed that all of the daily measures had sufficient within-person variability to make withinperson analyses feasible. Moreover, the means on each scale were sufficiently far from either endpoint so that floor and ceiling effects were not considerations.

Within this multilevel framework it was not possible to compare means of single items, that is, differences in how often people reported using the different strategies. To do this, within-person means for each of the four strategies were calculated, and these means were compared with a series of repeated measures analysis of variance. These analyses found that reappraisal was used more often than suppression for both positive and negative emotions and that suppression of positive emotions was the least frequently used strategy of the four $(p s<.01)$. (NB: These within-person means are identical to the estimates presented in Table 1.)

These analyses were followed by multilevel analyses that included all four emotional regulation measures as independent variables at Level 1 (the within-person level) and the other daily measures as dependent variables. The model is below:

$$
y_{i j}=\beta_{0 j}+\beta_{1 j} \text { PosReap }+\beta_{2 j} \text { NegReap }+\beta_{3 j} \text { PosSupp }+\beta_{4 j} \text { NegSupp }+r_{i j}
$$

In these analyses $y_{i j}$ is a dependent measure for person $j$ on occasion $i$, and $\beta_{1 j}$ through $\beta_{4 j}$ are coefficients representing relationships between $y$ and the four measures of regulation (reappraisal of positive and negative emotions and suppression of positive and negative emotions). Each measure of regulation was entered group mean centered, and, initially, all coefficients were modeled as random. Coefficients were fixed following the guidelines offered by Nezlek (2001).

The results of these analyses are presented in Table 2 . The coefficients in the table are mean unstandardized coefficients representing relationships between regulation and the various dependent measures. For example, in the analysis of positive active affect, the coefficient for reappraisal of positive emotions was .10. This means that for each increase of 1.0 in reappraisal of positive emotions, positive active affect increased .10. When evaluating these coefficients, it is important to keep in mind that each of the coefficients represents a relationship that was adjusted for the other three because all four regulation strategies were included in each analysis. Overall, and consistent with our expectations, the results indicate 
Table 2

Relationships Between Emotional Regulation and Other Daily Measures

\begin{tabular}{lccccc}
\hline & \multicolumn{2}{c}{ Reappraisal } & & \multicolumn{2}{c}{ Suppression } \\
\cline { 2 - 3 } \cline { 5 - 6 } & Positive & Negative & & Positive & Negative \\
\hline Positive active affect & $.10^{* * * *}$ & .04 & & $-.08^{* * * *}$ & -.00 \\
Positive deactive affect & $.08^{* * *}$ & .00 & & $-.05^{*}$ & .00 \\
Negative active affect & -.01 & .01 & & $.07^{* * *}$ & .01 \\
Negative deactive affect & -.01 & .00 & & $.09^{* * *}$ & $.04^{*}$ \\
Self-esteem & $.07^{* * *}$ & -.01 & & $-.07^{* * *}$ & $-.04^{*}$ \\
Cognitive triad & $.06^{* * *}$ & $.04^{*}$ & & $-.08^{* * * *}$ & -.01 \\
\hline
\end{tabular}

Note: Coefficients are unstandardized.

$* p<.05 . * * p<.01 . * * * p<.001$.

that emotional reappraisal was associated with positive outcomes, whereas emotional suppression was associated with negative outcomes.

The extent to which people reappraised situations to increase positive emotions was positively related to self-esteem, psychological adjustment, and positive affect (both active and passive, PA and PD). This means that on days when people used more positive reappraisal, they had higher self-esteem, they were better adjusted, and they experienced more positive emotions, both active (e.g., happy) and deactive (e.g., content). Similarly, the more participants reappraised negative emotions, the higher their psychological adjustment was.

In contrast, suppressing the expression of positive emotions was associated with decreased self-esteem, decreased psychological adjustment, and decreased PA and PD, and greater ND (e.g., sadness) and greater NA (e.g., anxiety). Similarly, suppressing the expression of negative emotions was associated with decreased self-esteem and greater ND. Overall, the coefficients in Table 2 suggest the broad conclusions we discuss here.

Positive emotions appear to be more susceptible to regulation than negative emotions. Only 1 of 8 coefficients between the regulation of negative emotions and emotional experience was significant, whereas 6 of 8 coefficients between the regulation of positive emotions and emotional experience were significant. Moreover, 
reappraisal (of positive emotions) appears to have generally positive consequences (greater experienced PA and PD and increased adjustment and self-esteem), whereas suppression appears to have primarily (if not exclusively) negative consequences (decreased PA, PD, adjustment, and self-esteem and increased NA and ND).

Given these relationships, we were interested in knowing if relationships between regulation and adjustment were mediated by relationships between regulation and affect. Additional analyses found that all measures of affect were significantly related to self-esteem and to psychological adjustment (all $p s<.05$ ). Given this, the analyses of self-esteem and adjustment measures were repeated with PA and PD as independent measures (along with the four regulatory strategies) and then again with NA and ND as independent measures.

These analyses suggested that positive and negative emotions played different roles in mediating the effects of, respectively, reappraisal and suppression on psychological adjustment. The analyses suggest that positive emotions mediate the effects of reappraisal on psychological adjustment and self-esteem, whereas negative emotions mediate the relationship between suppressing positive emotions and psychological adjustment and self-esteem. ${ }^{1}$

In particular, positive emotions mediated the effects of reappraisal on psychological adjustment and self-esteem. When PA and PD were included as predictors along with all four emotional regulation strategies, PA and PD remained significant predictors of self-esteem (all $p s<.001$ ), whereas reappraising positive and negative emotions were not significantly related to either self-esteem or psychological adjustment (all $p s>.12$ ). In contrast, negative emotions mediated the effects of the suppression of positive emotions. For both self-esteem and psychological adjustment, the coefficient for suppressing positive emotions became nonsignificant (all $p \mathrm{~s}>.07$ ) when NA and ND were taken into account, whereas NA and ND were significantly related to both self-esteem and adjustment (all $p s<.001$ ). The only relationship between emotion regulation and psychological adjustment and self-esteem that was not mediated by positive or negative

1. Recently, Bauer, Preacher, and Gil (2006) proposed a method to evaluate mediation in multilevel models, and one of the advantages of their technique is the ability to examine partial mediation. Given the patterns of mediation we found, there was no need to use these more complicated techniques. 
emotions was the relationship between suppressing negative emotions and self-esteem, which remained significant after controlling for positive or negative emotions ( $p s<.05$ and $<.01$, respectively).

\section{Sex Differences}

Although sex differences in emotional regulation have not received much attention either theoretically or empirically, on an exploratory basis, we examined sex differences in relationships between daily emotional regulation and daily emotional experience. This was done by including a contrast-coded variable $(1=$ women, $-1=$ men $)$ at Level 2 for all of the coefficients estimated in the analysis of each variable. The results of these analyses were reasonably clear. Sex moderated the relationship between the suppression of positive emotions and psychological adjustment $\left(\gamma_{41}=-.06, p<.01\right)$, PD $\left(\gamma_{41}=-.05, p<.05\right)$, PA $\left(\gamma_{41}=-.08, p<.01\right)$, NA $\left(\gamma_{41}=.03\right.$, $p=.08)$, and ND $\left(\gamma_{41}=.05, p<.01\right)$. In each case, the estimated coefficients indicated that suppressing positive emotions had more negative consequences for women than it did for men. Similarly, sex moderated the relationship between reappraising negative emotions and PA $\left(\gamma_{21}=-.06, p=.01\right)$. The relationship between PA and reappraising negative emotions was stronger for women than it was for men. There was only one instance for which men had a stronger relationship than women between regulation and a daily measure, selfesteem, and the suppression of negative emotions $\left(\gamma_{41}=.04, p<.05\right)$. Predicted values suggested that this relationship was functionally 0 for women and was -.08 for men.

\section{DISCUSSION}

\section{How Do People Try to Regulate Their Emotional Experience?}

In terms of use, the present results suggest that in their everyday lives, people try to regulate their emotions more through reappraisal than through suppression, a difference that seems to be larger for positive emotions than it is for negative emotions. Moreover, suppressing positive emotions was used much less than the other three strategies. Such differences make sense when the goals of each strategy are considered. The goal of reappraising positive emotions is to increase experienced positive emotion, and the goal of reappraising 
negative emotions is to decrease experienced negative emotion. Both of these goals represent outcomes that are immediately or directly rewarding and relevant to the person who is doing the reappraisal.

In contrast, the rewards of suppression are not as immediate or as directly relevant to the individual as are the goals of reappraisal. Suppression involves the display of emotions, and, therefore, suppression is more interpersonally focused than reappraisal, which is more intrapersonally focused. Although there may be a more distal goal of changing internal states by changing their expression (following the logic of the feedback hypothesis, e.g., Izard, 1993), the immediate goal of suppression is display, display that may often be for others (e.g., Russell, Bachorowski, \& Fernandez-Dols, 2003).

Within this context, suppressing the expression of positive emotions probably serves fewer goals than suppressing negative emotions. Moods and emotions can be contagious (e.g., Hatfield, Cacioppo, \& Rapson, 1993), and reducing the expression of negative emotions may reduce the negative emotions others experience, which, in turn, may reduce the negative emotions the person him- or herself experiences. In contrast, reducing the expression of positive emotions may reduce the positive emotions others experience, which, in turn, may reduce the positive emotions the person him- or herself experiences.

Reducing the expression of positive emotions may diminish the rewards people derive from interaction, a reduction that may inhibit people from suppressing positive emotions. Certainly, there may be times when suppressing positive emotions serves a purpose, e.g., controlling one's laughter when laughing is inappropriate. Moreover, there may be times when people suppress the expression of emotion in an explicit attempt to regulate their own emotional experience - for example, "I have got to stop crying so I can think straight about this problem," or "This all seems to be too good to be true. I need to be realistic about this." Nevertheless, our results suggest that reducing the expression of negative emotions is not that effective and that reducing the expression of positive emotions may have undesirable consequences, a possibility discussed below.

\section{How Is Emotion Regulation Related to Emotional Experience?}

The within-person covariation between emotional regulation and daily affective experience suggests that people are modestly successful in regulating their positive emotions. Within-person relationships 
between emotional experience and the regulation of positive emotions were stronger than the relationships between emotional experience and the regulation of negative emotions. For example, the use of positive reappraisal was associated with increased positive affect, both PA and PD, whereas negative reappraisal was not related to affective experience.

Whereas reappraisal was generally related to positive outcomes, regulation by suppression was generally related to undesirable outcomes. We found that suppressing the expression of positive emotions was associated with decreased positive experience and increased negative emotional experience. This occurred despite the fact that suppression does not concern controlling emotional experience per se. In contrast, suppressing negative emotions was not related to the experience of $\mathrm{PA}, \mathrm{PD}$, or NA, but it was positively related to the experience of ND.

These results suggest that suppressing positive emotions affects emotional experience more than suppressing negative emotions, and they agree partially with those of Gross and John (2003) who noted "one intriguing point of asymmetry... . whereas the suppressing of negative emotions left intact the experience of negative emotion, suppressing positive emotions decreased the experience of these emotions" (p. 349). Our data suggest that, at least on a daily basis, people's affective experience is (at most) only weakly related to, and is certainly not improved by, suppressing negative feelings. If anything, attempts to suppress the expression of negative emotions are associated with increases in deactive negative emotions. In general, the results suggest that it is more difficult (at least in terms of reappraisal and suppression) to regulate negative emotions than positive emotions. Most probably, the less frequent and more intense nature of most negative emotions contributes to this difference. Nevertheless, despite the relative lack of effectiveness, people still engage in efforts to change their negative emotions relatively frequently.

The foregoing discussion has tacitly assumed a causal link from regulation to experience; however, the opposite causal relationship needs to be considered. Our findings can also be interpreted to suggest that the experience of certain emotions facilitates or increases the use of certain emotion regulation strategies. Of particular interest was the finding that the experience of positively valenced emotions and psychological adjustment and self-esteem were related 
to increased use of reappraisal. In line with the broaden-and-build theory (Fredrickson, 2001), this suggests that positive emotions allow a person to consider more interpretations of an event, to broaden their view of what happened and, consequently, to be more successful in reevaluating their circumstances.

\section{How Is Emotional Regulation Related to Psychological Adjustment and Self-Esteem?}

The present study also concerned within-person relationships between emotional regulation and psychological adjustment and selfesteem. As expected, zero-order relationships between reappraisal and psychological adjustment and self-esteem were generally positive, whereas zero-order relationships between suppression and psychological adjustment and self-esteem were negative. In addition to these differences in direction, additional analyses suggested a very different role for affective processes in these relationships.

Follow-up analyses suggested that relationships between reappraisal and psychological adjustment and self-esteem were due in large part to the associated increases in positive affect. When experienced positive affect (PA and PD) was included as a predictor, the existing relationships between reappraisal and psychological adjustment and self-esteem became nonsignificant. Given the demonstrated within-person, day-level relationships between positive affect and psychological adjustment and self-esteem (e.g., Nezlek, 2005), such a change makes sense. The use of positive reappraisal led to increased positive affect, which, in turn, led to increased psychological adjustment and self-esteem.

In contrast, the negative relationship between the suppression of positive emotions and psychological adjustment was mediated by negative emotions. When experienced negative affect was included as a predictor, relationships between positive suppression and psychological adjustment and self-esteem that were significant became nonsignificant. The changes in adjustment and self-esteem associated with suppressing the expression of emotions appeared to be related to changes in negative affect.

Our results suggest that reappraisal is beneficial because it seems to generate positive feelings that increase self-esteem and increase well-being defined within the context of Beck's model of depression. In contrast, suppressing the expression of positive emotions is not 
beneficial (and possibly harmful) because it is accompanied by negative feelings that have detrimental effects on self-esteem and adjustment. Vice versa, our results may also indicate that increased psychological adjustment and self-esteem foster positive reappraisal through the experience of positive emotions and may decrease the suppression of positive emotions because of decreases in negative experiences. In any case, our results suggest that reappraisal and suppression are differentially related to psychological adjustment and self-esteem. Reappraisal is related via positive emotions, whereas suppression (or, at least, the suppression of positive emotions) is related via negative emotions.

Our results also found that suppressing positive emotions was more strongly associated with decreases in adjustment and selfesteem and positive emotions and increases in negative deactive emotions for women compared to men. In this respect, previous research has consistently found that women smile (i.e., express positive emotion) more than men, perhaps because of gender stereotypes and social roles (LaFrance, Hecht, \& Levy Paluck, 2003). Our results suggest that when women do not conform to these stereotypes or roles (i.e., when they suppress the expression of positive emotions), this has more negative consequences for their adjustment than it does for men, for whom the expression of positive emotion may be a less important part of sex role expectations.

\section{Conceptualizing Different Types of Emotional Regulation}

In terms of the conceptualization and measurement of emotional regulation, the present results suggest that it may be useful to distinguish the regulation of positive and negative emotions, at least at the state level, particularly for suppression. The differences between the regulation of positive and negative emotions found in the present study contrasts with the fact that the popular measure of trait regulation offered by Gross and John (2003) does not distinguish these two, although items on the measure concern the regulation of positive and negative emotion. These differences may reflect differences in the state and trait levels of these constructs, a possibility discussed by Tennen at al. (2000). The differences may also reflect limitations of the item set used by Gross. The variance shared at the trait level by the positive and negative reappraisal items and that shared by the 
suppression items may be great enough to lead to a two factor solution that does not distinguish the regulation of positive and negative emotions.

To our knowledge, there is only one published study on the daily self-regulation of emotions, Kashdan and Steger (2006). Direct comparisons with their results and ours are difficult because of differences between the studies in measures that were used and differences in the topics of interest. In terms of regulation strategies, we distinguished the regulation of positive and negative emotions, whereas Kashdan and Steger did not, and Kashdan and Steger did not report results involving emotional reappraisal. In terms of emotional experience, we distinguished active and deactive emotions, whereas Kashdan and Steger did not. Moreover, they did not report simple, zero-order, within-person relationships between daily emotional regulation and daily emotional experience. Nevertheless, their conclusion that emotional suppression was negatively related to experienced positive emotions (albeit with some caveats) is consistent with our results.

\section{Limitations and Conclusions}

Certainly, our conclusions are limited by the sample and methods at hand. Another important limitation lies with the cross-sectional design of the study. The data we collected did not provide a firm basis for conclusions about causal relationships between self-regulation and our other measures. On the basis of our study, we cannot conclude whether or to what extent the relationships we found reflect the fact that emotional regulation affects experience and psychological adjustment, or that emotional experience and psychological adjustment drives the use of particular types of emotion regulation. Nonetheless, our results provide ecological corroboration of previous laboratory findings, which have largely assumed a causal relationship between emotional regulation and other outcomes, and, as such, our results provide useful information about which types of emotion regulation are related to emotional experience and psychological adjustment in daily life.

Moreover, there are limitations associated with the methods and measures. For example, we relied upon end-of-day reports regarding daily emotional states. It is possible that such reports did not reflect how people felt that day but did reflect (despite instructions to the 
contrary) the strongest feeling people had during a day or how they were feeling at the end of the day or how they were feeling when they provided the rating. We also examined ways of regulating emotions suggested by Gross's model and ignored others such as relaxation or distraction.

Our findings may also be limited by our use of single-item measures of emotional regulation and by possible limitations of our conceptualization of suppression. For example, individuals who do not experience a particular type of emotion (either often or strongly or both) will not have the opportunity to suppress such an emotion. Although our state measures were worded very similarly to the popular trait measure used by Gross and colleagues, and similar to the trait measure, our items referred to suppression when emotions had occurred, such a possibility cannot be discounted.

Regardless, the current findings are important because they show how different emotion regulation strategies are used in daily life and how emotional regulation is related to emotional experience and psychological adjustment. Our results suggest that people reappraise emotions more often than they suppress them. Moreover, it appears that the use of reappraisal is related to increased positive affect, psychological adjustment, and self-esteem, whereas suppressing the expression of positive emotions is related to decreased levels of positive emotions, psychological adjustment, and self-esteem. We also found that emotion regulation was only weakly related to the experience of negative emotions, suggesting that negative emotions are harder to regulate than positive emotions. Alternatively, negative emotions may somehow disable the use of emotion regulation more than positive emotions, despite the fact that people attempt to regulate negative emotions just as often (or more in the case of suppression) than they attempt to regulate positive emotions.

Theoretically speaking, the present results suggest that the regulation of positive and negative emotions needs to be distinguished, at least at the within-person level. The fact that experienced positive emotions mediated the relationship between reappraisal and psychological adjustment and self-esteem further suggests that distinguishing within- and between-person processes may be particularly important when studying emotional regulation. Practically speaking, the present results agree with previous research in suggesting that reappraising emotions is associated more with beneficial psychological states than suppressing emotions. 


\section{REFERENCES}

Bauer, D. J., Preacher, K. J., \& Gil, K. M. (2006). Conceptualizing and testing random indirect effects and moderated mediation in multilevel models: New procedures and recommendations. Psychological Methods, 11, 142-163.

Beck, A. T. (1972). Depression: Causes and treatments. Philadelphia: University of Pennsylvania Press.

Cacioppo, J. T., \& Gardner, W. L. (1999). Emotion. Annual Review of Psychology, 50, 191-214.

Davidson, R. J., Pizzagalli, D., Nitschke, J. B., \& Putnam, K. (2002). Depression: Perspectives from affective neuroscience. Annual Review of Psychology, 53, 545-574.

Davidson, R. J., Putnam, K. M., \& Larson, C. L. (2000). Dysfunction in the neural circuitry of emotion regulation-A possible prelude to violence. Science, 289, 591-594.

Diener, E., Suh, E. M., Lucas, R. E., \& Smith, H. L. (1999). Subjective well-being: Three decades of progress. Psychological Bulletin, 125, 276-302.

Dodge, K. A. (1989). Coordinating responses to aversive stimuli: Introduction to a special section in the development of emotion regulation. Developmental Psychology, 25, 339-342.

Eisenberg, N. (2000). Emotion, regulation, and moral development. Annual Review of Psychology, 51, 665-697.

Feldman Barrett, L., \& Russell, J. A. (1998). Independence and bipolarity in the structure of current affect. Journal of Personality and Social Psychology, 74, 967-984.

Fredrickson, B. L. (2001). The role of positive emotions in positive psychology. The broaden-and-build theory of positive emotions. American Psychologist, 56, 218-226.

Gross, J. J. (1998). The emerging field of emotion regulation: An integrative review. Review of General Psychology, 2, 271-299.

Gross, J. J. (2002). Emotion regulation: Affective, cognitive, and social consequences. Psychophysiology, 39, 281-291.

Gross, J. J., \& John, O. P. (2003). Individual differences in two emotion regulation processes: Implications for affect, relationships, and well-being. Journal of Personality and Social Psychology, 85, 348-362.

Gross, J. J., \& Levenson, R. W. (1997). Hiding feelings: The acute effects of inhibiting negative and positive emotion. Journal of Abnormal Psychology, 106, 95-103.

Hatfield, E., Cacioppo, J. T., \& Rapson, R. L. (1993). Emotional contagion. Current Directions in Psychological Science, 2, 96-99.

Izard, C. E. (1993). Four systems of emotion activation: Cognitive and noncognitive processes. Psychological Review, 100, 68-90.

Kashdan, T. B., \& Steger, M. F. (2006). Expanding the topography of social anxiety. Psychological Science, 17, 120-128.

LaFrance, M., Hecht, M. A., \& Levy Paluck, E. (2003). The contingent smile: A meta-analysis of sex differences in smiling. Psychological Bulletin, 129, 305-334. 
Lerner, J. S., \& Keltner, D. (2001). Fear, anger, and risk. Journal of Personality and Social Psychology, 81, 146-159.

Nezlek, J. B. (2001). Multilevel random coefficient analyses of event and interval contingent data in social and personality psychology research. Personality and Social Psychology Bulletin, 27, 771-785.

Nezlek, J. B. (2005). Distinguishing affective and non-affective reactions to daily events. Journal of Personality, 73, 1539-1568.

Nezlek, J. B. (2007). Multilevel modeling in research on personality. In R. Robins, R. C. Fraley, \& R. Krueger (Eds.), Handbook of research methods in personality psychology (pp. 502-523). New York: Guilford.

Panksepp, J. (1998). Affective neuroscience. The foundation of human and animal emotions. New York: Oxford University Press.

Raudenbush, S. W., \& Bryk, A. S. (2002). Hierarchical linear models (2nd ed.). Newbury Park, CA: Sage Publications.

Raudenbush, S. W., Bryk, A. S., Cheong, Y. F., \& Congdon, R. (2000). HLM6. Chicago: Scientific Software.

Rosenberg, M. (1965). Society and the adolescent self-image. Princeton, NJ: Princeton University Press.

Russell, J. A., Bachorowski, J., \& Fernandez-Dols, J.-M. (2003). Facial and vocal expressions of emotion. Annual Review of Psychology, 54, 329-349.

Taylor, S. E. (1991). Asymmetrical effects of positive and negative events: The mobilization-minimization hypothesis. Psychological Bulletin, 110, 67-85.

Tennen, H., Affleck, G., Armeli, S., \& Carney, M. A. (2000). A daily process approach to coping. Linking theory, research, and practice. American Psychologist, 55, 626-636. 
